\title{
ABANDONOS TERAPÉUTICOS REGISTRADOS EN EL ÁMBI- TO DE LA HIPERACTIVIDAD Y LOS TRASTORNOS DEL COMPORTAMIENTO. INFLUENCIA DE VARIABLES INDIVI- DUALES Y FAMILIARES
}

\section{Inmaculada Moreno García Jose Antonio Lora Muñoz}

Departamento de la Personalidad, Evaluación y Tratamientos Psicológicos. Facultad de Psicología. Universidad de Sevilla

\section{RESUMEN}

Se analiza la influencia del trastorno infantil (trastorno por déficit de atención con hiperactividad y trastomo negativista desafiante), los métodos de manejo conductual adoptados por los padres y su estado de salud en la continuidad o abandono terapéutico por parte de los niños previamente diagnosticados. En el estudio han participado 62 niños (5-12 años). Tras el diagnóstico, el registro de la asistencia/ausencia de los pacientes a las sesiones de tratamiento programadas permitió diferenciar tres grupos: a) niños que rehusaron la terapia recomendada, b) menores que, tras varias sesiones, interrumpieron el tratamiento y c) pacientes que finalizaron el programa de intervención. Los resultados muestran influencia significativa del trastorno diagnosticado y los métodos de manejo conductual adoptados por los padres en los abandonos terapéuticos, cuya

Correspondencia: Departamento de Personalidad, Evaluación y Tratamientos Psicológicos. Facultad de Psicología. Universidad de Sevilla. C/ Camilo José Cela, s/n. 41018. Sevilla (España. Teléfono: 954556929 . Fax: 954557807. 
tasa se estima en el $37 \%$ de los casos, similar a los hallazgos de las investigaciones sobre el tema.

Palabras clave: TERAPIA INFANTL, CONTINUIDAD Y ABANDONOS TERAPÉUTICOS, VARIABLES PERSONALES Y FAMILIARES.

\section{SUMMARY}

Throughout this work we are going to analyze the influence of the infantile disorder (deficit attention-hyperactivity disorder and oppositional defiant disorder), the methods of behavior management used by the parents and their condition in the continuity or therapeutic drop-out on the part of the children previously diagnosed. 62 children have participated (5-12 years) in this study. After the diagnosis, the register of the presencel absence of patients to the programmed sessions of treatment allowed us to differentiate three groups: a) children who refused the recommended therapy, b) children who, after several sessions, interrupted the treatment and c) patients who ended up the intervention program. The results show the significant influence of the diagnosed disorder and the methods of behavioural management adopted by the parents in the therapeutic drop-outs, whose rate is estimated in $37 \%$ of the cases, similar to the results of published investigations on the subject.

Key wOrds: INFANCY THERAPY, TREATMENT CONTINUITY AND DROPOUTS, PERSONAL AND FAMILIAR VARIABLES.

\section{INTRODUCCIÓN}

Los trabajos que, en el ámbito infantil, indagan sobre los motivos de consulta o problemas que causan la mayor demanda de atención clínica revelan que los trastornos del comportamiento, hiperactividad, 
oposicionismo y agresividad centran la atención prioritaria tanto en el ámbito de la investigación como en el de la práctica clínica (Kazdin, Siegel y Bass, 1990). Desde la perspectiva terapéutica las investigaciones realizadas, respecto a estos trastornos, han permitido identificar tratamientos eficaces (Nathan, Gorman y Salkind, 2002) e investigar los factores que median los resultados clínicos obtenidos (Kazdin, 2003). En consonancia con estas actuaciones y debido en parte a la preocupación actual por determinar la eficacia y relación coste-beneficio de las intervenciones clínicas se aprecia cierto interés por examinar los abandonos del tratamiento infantil (Moreno, 2005).

La investigación sobre interrupciones y ceses injustificados en las terapias infantiles se desarrolla actualmente teniendo en cuenta las siguientes condiciones específicas: a) Los niños y jóvenes que han sido diagnosticados presentan alguno de los trastornos del comportamiento diferenciados y la propuesta de intervención es consecuente con tal diagnóstico, b) Existe demanda de ayuda terapéutica planteada por los padres 0 adultos responsables del menor afectado y c) En buena parte de los casos se recomiendan tratamientos que, según las investigaciones clínicas precedentes, cuentan con evidencia empírica sobre sus resultados. Sin embargo, pese a la convergencia de tales factores: demanda terapéutica, diagnóstico formulado en base a criterios diagnósticos consensuados y propuesta de tratamiento eficaz, un porcentaje considerable de pacientes infantojuveniles no llega a iniciar la terapia, o la abandonan tras varias sesiones sin haber obtenido los resultados clínicos esperados.

No cabe duda que esta circunstancia repercute de forma adversa en los propios afectados que, al interrumpir injustificadamente la terapia, no se benefician de los efectos asociados al tratamiento recomendado, a diferencia de lo que se observa en aquellos individuos que completan la intervención y se aprovechan de los beneficios terapéuticos derivados de la misma. Asimismo, los abandonos terapéuticos constituyen un obstáculo y limitan las posibilidades de hacer efectivos los servicios de atención psicológica a los pacientes infantiles que la requieren y a los padres que habitualmente la demandan. Por otro lado, desde la perspectiva de la investigación clínica, las interrupciones injustificadas y ceses prematuros del tratamiento por parte de algunos sujetos participantes en los estudios científicos 
inciden de forma adversa en el alcance y significación de los hallazgos obtenidos pues, es sabido que tal circunstancia altera la composición de los grupos de sujetos, introduce sesgos en el muestreo y limita la generalización de los resultados (Kazdin, Holland y Crowley, 1997).

En cualquier caso, las investigaciones realizadas sobre el tema se han ocupado, entre otras, de las siguientes cuestiones (Wierzbicki y Pekari, 1993; Kazdin y Wassell, 1994; Kazdin y Mazurick, 1994; Kazdin, Holland y Crowley, 1997; Salvador y Melgarejo, 2000; Brown, 2003 y Kraim, 2004): a) Interés por delimitar conceptualmente el fenómeno a partir de la discusión en torno a los conceptos de permanencia, continuidad, abandono, $\theta$ interrupción de la terapia b) Preocupación por el alcance y dimensión que adquieren en la práctica e investigación clínica los abandonos terapéuticos, c) Análisis de las variables y factores que parecen estar relacionadas y predecir las interrupciones injustificadas del tratamiento.

El interés por consensuar el concepto de abandono terapéutico es unánime si se tiene en cuenta que Wierzbicki y Pekari (1993) hallaron diferencias significativas respecto a las tasas de abandonos registrados dependiendo de la concepción adoptada en los distintos trabajos analizados al respecto. En un intento por clarificar el panorama existente, estos autores sistematizaron en tres propuestas las opciones manejadas hasta entonces: a) considerar abandono cuando el paciente no ha completado la totalidad del programa terapéutico diseñado al efecto, b) conceptualizar la cuestión a partir del número de sesiones terapéuticas realizadas y c) estimarlo en función del juicio del propio terapeuta. Kazdin, Holland y Crowley, (1997) y Kazdin y Wassell (1998) tratan de simplificar la cuestión diferenciando entre abandono terapéutico, conceptualizado como interrupción prematura del tratamiento a partir de la decisión adoptada por los padres, sin tener el cuenta el criterio del terapeuta y tratamiento completo referido a aquellos casos que finalizan el tratamiento coincidiendo, en tal sentido, el terapeuta y la familia.

Sin embargo, la polémica no se agota en esta distinción pues el debate queda planteado en torno a la idoneidad e inconveniencia de ampliar el concepto de abandonos terapéuticos, extendiéndolo a aquellos pacientes que no inician la terapia recomendada, acorde 
con el diagnostico previamente establecido. A propósito de esta cuestión, Garfield (1989), sensible con los planteamientos de Levitt (1971), propone el término rehusar para referirse a los pacientes que cumplen las siguientes condiciones: a) han sido diagnosticados, b) han aceptado el tratamiento recomendado, pero c) no han asistido a ninguna sesión formal del mismo.

Respecto a la tasa de abandonos, se estima que, en general, entre el $30 \%$ y $60 \%$ de los pacientes interrumpen prematuramente el tratamiento prescrito (Kazdin y Wassell, 1998). Los resultados del metaánalisis realizado por Wierzbicki y Pekari (1993) a partir de 125 trabajos publicados entre 1974 y 1990 que contaban con muestras de adultos, niños y mixtas, revelaron que el $47 \%$ (rango $42,9 \%$ $50,82 \%$ ) de los pacientes con indicaciones de tratamiento abandonaron antes de dar por concluida la terapia. En este trabajo también quedó de manifiesto la necesidad de investigar la cuestión por separado, atendiendo a la edad de los destinatarios de la intervención, tal como antes habían concluido Pekarik y Stephenson (1988) y Pekarik (1991). En el ámbito infantil los datos obtenidos revelan que entre $40-60 \%$ de los niños y adolescentes diagnosticados que requieren tratamiento abandonan prematuramente la terapia (Kazdin, 1996). Kazdin y Wassell (1998) hallaron que el $38 \%$ de los menores, con edades comprendidas entre 3 y 13 años, remitidos a tratamiento por mostrar comportamientos antisociales, cesaron injustificadamente la intervención. Pelham y Murphy (1986) observaron que la mitad de los padres interrumplan la aplicación de los tratamientos conductuales recomendados a sus hijos pese a la opinión contraria del terapeuta y Lynn (2004), subrayaba que el $50 \%$ de las familias no continuaba con las prescripciones clínicas recomendadas tanto en el tratamiento farmacológico como en las terapias psicológicas empleadas en estos casos.

Por otro lado, se ha analizado la influencia y el valor predictivo que mantienen, respecto a esta cuestión, los siguientes factores: a) variables demográficas (sexo, edad, etnia, nivel educativo y nivel socioeconómico), b) factores individuales (disfunción clínica diagnosticada, severidad del trastorno, historia previa de tratamientos), c) familiares (estrés y psicopatologia de los padres expectativas sobre el tratamiento, etc.), y variables relacionadas $d$ ) con el propio terapeuta 
(sexo, raza, experiencia, etc.) y e) con el tratamiento recibido (duración de la terapia, contenido técnico y obstáculos surgidos durante el curso del tratamiento). Con independencia de si los pacientes son adultos 0 niños, las investigaciones publicadas muestran que los recursos socioeconómicos, las diferencias étnicas y el nivel educativo parecen influir significativamente en los abandonos terapéuticos estudiados (Wierzbicki y Pekari (1993), Kazdin, Holland y Crowley, (1997), Lynn (2004). Específicamente, en el ámbito de los trastornos del comportamiento Armbruster y Kazdin (1990) hallaron que variables familiares (desventaja socioeconómica, prácticas educativas adversas), factores relacionados con los padres (estrés, acontecimientos vitales estresantes, psicopatología parental e historia de comportamientos antisociales) y con el propio paciente (severidad y cronicidad del trastorno, relaciones conflictivas entre iguales) constituían factores de riesgo para predecir interrupciones tempranas de los tratamientos administrados a niños con edades comprendidas entre 4 y 13 años. Resultados similares también han sido aportados por Webster-Stratton y Hammond (1990). A estos factores hay que añadir las atribuciones que mantienen los padres sobre los problemas de su hijos (Corcoran e Ivery, 2004) y los obstáculos que plantean en relación al tratamiento infantil y sus expectativas acerca del éxito del mismo. Kazdin, Holland y Crowley (1997) observaron que los obstáculos prácticos esgrimidos por los padres para implicarse en la terapia junto a su percepción adversa del tratamiento infantil recomendado, y la escasa relación que habían mantenido con el terapeuta contribuían significativamente a los abandonos terapéuticos registrados en el estudio en el que participaron 242 familias. Sin embargo, en el trabajo de Corkum, Rimer y Schachar (1999), quedó de manifiesto que la continuidad en el tratamiento administrado a 81 niños hiperactivos (5-12 años) no mantenía relación alguna ni con el nivel de conocimiento de los padres sobre este trastorno ni tampoco con sus opiniones sobre la terapia seleccionada. En todo caso, la influencia de las expectativas y aceptación de la terapia infantil por parte de los progenitores se ha investigado atendiendo a los procedimientos conductuales (Jones, Eyberg, Adams y Bogas, 1998) por un lado, y a la naturaleza farmacológica o psicológica del tratamiento, por otro (Lynn, 2004). 
Teniendo como marco de referencia las cuestiones antes mencionadas en el trabajo que se expone a continuación el grupo de niños que ha finalizado el tratamiento está integrado por los pacientes que han concluido el programa de intervención propuesto, habiéndose obtenido resultados evaluados a partir de los siguientes indicadores: a) cambios conductuales estimados en base a los registros iniciales, b) informes coincidentes de padres, profesores y terapeuta y c) normalización, atendiendo a los parámetros de su grupo de referencia, en cuanto a rendimiento académico. Asimismo, hemos diferenciado entre interrupciones del tratamiento y rehusar la terapia, asumiendo en este caso el concepto propuesto por Garfield (1989). No obstante, dado que no se disponen de datos sobre el alcance real que el tratamiento iniciado ha generado en los niños que han interrumpido la terapia, se ha optado por considerar ambos grupos (interrumpir y rehusar) como abandonos terapéuticos.

Así pues, esta investigación tiene planteados los siguientes objetivos: i) Estimar los abandonos terapéuticos registrados en una muestra de niños diagnosticados con trastorno por déficit de atención con hiperactividad y trastorno negativista desafiante, ii) Analizar la influencia que en esta cuestión ejercen el trastorno infantil diagnosticado, el estado de salud de los padres valorado cuando éstos deciden plantear la demanda terapéutica y los métodos de manejo conductual adoptados por los progenitores. Por último, iii) se analiza la relación existente entre el trastorno infantil diagnosticado y los factores relacionados con los padres.

\section{MATERIAL Y MÉTODO}

\section{Muestra}

Han participado en la investigación niños remitidos a consulta, a iniciativa de los centros escolares, públicos y concertados de Sevilla y su provincia, en los que estaban escolarizados por problemas de hiperactividad, impulsividad, dificultades de atención, agresividad y oposicionismo. Se ha adoptado como criterio de inclusión cumplir los criterios diagnósticos del DSM-IV-TR (APA, 2000) referentes a los trastornos por déficit de atención y comportamiento perturbador. La 
muestra ha quedado constituida finalmente por 62 niños, 42 niños y 20 niñas, con edades comprendidas entre 5 y 12 años $0=8,1$, d.t. = $2,2)$ y sus padres, cuyo rango de edad oscilaba entre 25 y 48 años $(0=35,4$, d.t. $=6,1)$. 35 menores $(56,45 \%)$ cumplían los criterios del trastorno por déficit de atención con hiperactividad y $27(43,55 \%)$ los criterios del trastorno negativista desafiante.

\section{Instrumentos empleados}

Entrevista Clínica de Barkley (Barkley, Murphy y Bauermeister, 1998). Permite una exploración diagnóstica amplia, incluye los síntomas de los trastornos mentales más frecuentes que se diagnostican en la infancia basados en los criterios del DSM-IV e integra cuestiones relativas a la estructura e historial familiar, preocupaciones y procedimientos de manejo conductual, historial académico y terapéutico, así como competencias psicológicas-sociales del niño. Para valorar los métodos de manejo conductual que emplean los padres se empleó el formato adaptado que se incluye en el protocolo IMAT de evaluación del TDAH desarrollado en la Universidad de las Islas Baleares (Servera, Bornas y Moreno, 2001). De este modo se analizaron las siguientes variables: Cumplimiento/ejecución real de órdenes paternas, Acuerdo padre/madre en normas disciplina, establecimiento de amenazas reales, cumplimiento de castigos, utilidad normas disciplina, capacidad para establecer límites y eficacia de las estrategias conductuales adoptadas (padre/madre). Estas variables fueron valoradas cuantitativamente en una escala Likert de 1 a 5 . Respecto a las estrategias conductuales adoptadas por padre/madre se incluyeron las siguientes opciones de respuesta: nada, extinción, razonamiento, control de estímulos, castigo negativo y castigo positivo (verbal y físico). Finalmente, se consideraron las actividades realizadas de forma conjunta con el niño por ambos padres.

Adhd Rating Scale-IV (DuPaul, Power, Anastopoulos y Reid, 1998). Valora la presencia del trastorno por déficit de atención con hiperactividad en función de los criterios psiquiátricos del DSM-IV. Permite obtener tres valores de hiperactividad; puntuación global, referida a valoración general del trastorno y dos valores relativos al déficit atencional e hiperactividad-impulsividad, respectivamente. Esta 
prueba cuenta con baremos y se halla traducida al castellano (Barkley, 1997).

Listado de sintomas del TND en el DSM-IV-TR (APA, 2000). La utilización del listado de síntomas del DSM-IV para la evaluación del negativismo ha sido estimado como sistema idóneo para detectar la sintomatología de dicho trastorno, mostrando buenas propiedades psicométricas y adecuada utilidad clínica (Collett, Ohan y Myers, 2003). Su valoración se realiza a partir de cuatro alternativas de respuesta que oscilan entre "nunca o casi nunca" y "muy frecuentemente".

Inventario de Depresión de Beck (BDI) (Beck, Ward, Mendelson, Mock y Erbaugh, 1961). Este instrumento, empleado con similares objetivos en investigaciones sobre el tema (Kazdin y Wassell, 1998), cuenta con amplia y actualizada baremación en español y adecuadas propiedades psicométricas (Vázquez y Sanz, 1997).

Inventario de Ansiedad de Beck (BAl) (Beck, Epstein, Brown y Steer, 1988). Integrado por 21 ítems, es ampliamente utilizado para la valoración de los trastornos de ansiedad. Ha sido baremado y validado en distintas poblaciones (Osman, Hoffman, Barrios, Koper, Breitenstein, 2002), entre ellas, población española (Sanz y Navarro, 2003),

Cuestionario de Salud General de Golberg (GHQ-28) (Goldberg, 1978). ). Evalúa la Salud General (Salud Mental) autopercibida diferenciando entre malestar y salud psicológica. Detecta anomalías presentes en las dos últimas semanas anteriores a su aplicación y atiende a cuatro áreas destacadas: depresión, ansiedad, inadecuación social e hipocondria. Se trata de un cuestionario ampliamente utilizado y validado, entre otras, en población española (García, 1999).

Procedimiento: Tras el diagnóstico infantil se procedió a la evaluación de los padres al tiempo que se recogió información sobre aspectos relacionados con la convivencia familiar y la existencia de posibles conflictos conyugales. Posteriormente, se les informó del tratamiento más indicado para su hijo atendiendo al trastomo previamente identificado. Cuando los progenitores se mostraron conformes con la terapia propuesta se acordó la fecha para llevar a cabo la primera sesión de intervención. A partir de entonces la pauta de actuación seguida por los pacientes infantiles les diferenció respecto 
a las variables investigadas en este trabajo. Algunos niños no asistieron a la cita que marcaba el comienzo de la intervención, otros empezaron pero tras algunas sesiones interrumpieron la terapia y finalmente, la mayoría completó el tratamiento propuesto.

Las modalidades de tratamiento recomendadas y administradas a los niños participantes se seleccionaron de acuerdo con el trastorno diagnosticado y los hallazgos científicos que avalan su eficacia en el ámbito de los trastornos del comportamiento e hiperactividad. Se pusieron en práctica Entrenamiento a Padres, procedimientos de Manejo de Contingencias Ambientales y Entrenamiento en Habilidades Sociales, alternativas terapéuticas cuya eficacia, respecto a estos trastornos, está sostenida en la evidencia (Kazdin, 2003) y constituyen, especialmente, los programas de entrenamiento a padres el tratamiento de elección para intervenir en los problemas de conducta infantil (Remschmidt, 2003), Las intervenciones desarrolladas incluyeron específicamente el programa de entrenamiento a padres de niños desafiantes de Barkley (1997) adaptado y desarrollado en formato individualizado durante 5 sesiones de 60 minutos de duración. Ahora bien, todos los niños con trastorno por déficit de atención con hiperactividad, predominio déficit atencional, fueron tratados además, con alguna de las opciones terapéuticas encaminadas a mejorar el rendimiento académico que incluían preparación específica para ejecutar satisfactoriamente el trabajo escolar y entrenamiento del profesor (5 sesiones desarrolladas en dos meses). No obstante, la decisión de administrar, en estos casos, tratamiento con o sin entrenamiento cognitivo (aplicado individualmente entre 12 14 sesiones de 45 minutos de duración) se adoptó atendiendo a la severidad del problema atencional por un lado y a las deficiencias y limitaciones detectadas en cada niño respecto a los problemas de aprendizaje.

Análisis estadístico: Se han aplicado, para el análisis de las variables cuantitativas, las pruebas $t$ y $F$, realizando los análisis a posteriori mediante la prueba de Tukey. Asimismo, para las variables cualitativas se ha utilizado, mediante tablas de contingencia, el estadístico Chicuadrado de la razón de verosimilitud, analizando la significación específica de las comparaciones mediante la consideración de los residuos tipificados corregidos (R.T.C.). 


\section{RESULTADOS}

Según los criterios diagnósticos DSM-IV-TR (APA, 2000), 32,3\% $(n=20)$ de los niños participantes fueron diagnosticados con trastorno por déficit de atención con hiperactividad, tipo atencional, $24,2 \%$ ( $n=$ 15) Trastorno por déficit de atención con hiperactividad, tipo hiperactivoimpulsivo y tipo combinado, y $43,5 \%(n=27)$ trastorno negativista desafiante. De acuerdo con las investigaciones sobre el tema en las que queda de manifiesto la clara prevalencia de dos subtipos (déficit de atención y combinado) (Power, Costigan, Eịraldi y Leff, 2004). y teniendo en cuenta la distribución porcentual de los sujetos participantes, se decidió integrar en un único grupo a los niños que cumplian los criterios del subtipo hiperactivo/impulsivo y combinado.

Las puntuaciones obtenidas por los menores en las pruebas $A d h d$ Rating Scale-IV (DuPul, et al., 1988) y Listado de síntomas del TND se distribuyen como sigue: TDAH: Tipo déficit atencional $(0=17,44$; d.t. = 4,07), TDAH: Tipo hiperactivo- impulsivo $(0=19,20 ; d . t .=2,49)$, TDAH, Tipo combinado $(0=32 ;$ d.t. $=8,54)$, Trastorno negativista desafiante $(0=16,75 ;$ d.t. $=4,05)$.

A partir del inicio del tratamiento el registro de la asistencia/ausencia de los niños a las sesiones de tratamiento programadas permitió diferenciar, según la variable continuidad/abandono, tres grupos de pacientes: a) integrado por aquellos menores que no llegaron a iniciar el tratamiento propuesto (Rehusan) $(n=13,21 \%)$, b) configurado por los niños que, si bien comenzaron la intervención, abandonaron tras varias sesiones por decisión de sus padres $(n=10,16 \%), y c)$ el grupo más numeroso, formado por los menores que finalizaron el tratamiento recomendado $(n=39,63 \%)$.

Como podemos observar (Tabla 1) la relación entre el trastorno infantil diagnosticado previamente y la continuidad o abandono del tratamiento, valorada a través de la Chi-cuadrado de la Razón de verosimilitud, resulta estadísticamente significativa $\left(X^{2}{ }_{(4)}=12,37 ; p=\right.$ $0,015)$. Se aprecia asimismo, que la diferencia es relevante y significativa entre los niños diagnosticados con TDAH hiperactivo-cornbinado y los menores con trastorno negativista desafiante. Según los datos obtenidos, los niños con trastorro hiperactivo, tipo combinado, interrumpieron el tratamiento más de lo esperado $(33,3 \%$; R.T.C. $=2,08$; 
R.T.C. $> \pm 1,96)$ al tiempo que los menores con trastorno negativista finalizaron la terapia mayoritariamente $(85,19 \%$; R.T.C. $=3,19$; R.T.C. $> \pm 1,96)$.

Tabla 1. Relación entre las variables finalizar - abandonar el tratamiento $y$ el trastorno infantil diagnosticado

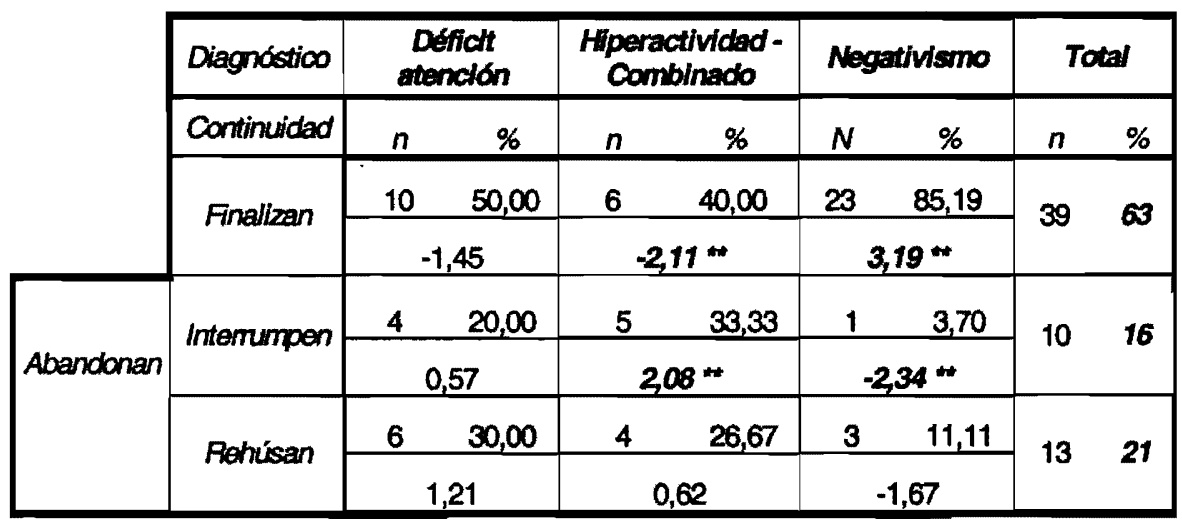

$\left(X^{2}=12,37 ; p=0,015\right)^{\star} \quad *(p<0,05) \quad * *($ R.T.C. $>+/-1,96)$

Con respecto a la salud psíquica de los padres, los resultados extraídos muestran, en relación al Cuestionario de Salud General de Golgberg (GHQ), las siguientes puntuaciones correspondientes a las cuatro escalas del instrumento y la puntuación global: síntomas somáticos $(0=10,65$, d.t. $=4,28)$, ansiedad $e$ insomnio $(0=12$, d.t. $=$ $4,82)$, disfunción social $(0=9,88$, d.t. $=2,83)$, depresión: $0=6,16$, d.t. $=$ $4,50)$ y puntuación global $(0=38,29$, d.t. $=14,09)$. Por lo que se refiere a los cuestionarios de depresión y ansiedad empleados, señalar que el $18 \%(n=11)$ de los adultos muestran depresión (fundamentalmente madres) $(0=28,6$; d.t. $=8,96)$ y el $26 \%(n=16)$ trastornos de ansiedad $(0=38,14$; d.t. $=7,3)$. Asimismo, el $18 \%(n=11)$ de los padres tenía problemas de pareja al comienzo de la intervención, mientras que en el $39 \%(n=24)$ restante no se han detectado ninguna de las alteraciones analizadas en el trabajo.

De acuerdo con los objetivos de esta investigación se analizó la relación observada entre la variable continuidad/abandono terapéutico y las puntuaciones obtenidas por los padres en el GHQ mediante un 
análisis de la varianza. Dicho estadístico aportó diferencias significativas $\left(F_{(2.59}=3,73 ; p=0,032\right)$ en la subescala tres, referente a disfunción social. El análisis a posteriori con la prueba de Tukey muestra que los padres de los menores que rehusaron al tratamiento obtuvieron, en ésta subescala, medias significativamente inferiores $(0=7,88$; d.t. $=2,59$ ) a los valores obtenidos por los progenitores de los niños que interrumpieron la terapia $(0=11,25$; d.t. $=2,50)$, quedando las puntuaciones de los padres cuyos hijos finalizaron en un rango intermedio $(p=0,024)$.

Asimismo, los datos obtenidos muestran que las alteraciones psicopatológicas detectadas en los padres se encuentran relacionadas de forma significativa $\left(X_{(3)}^{2}=11,27 ; p=0,011\right)$ con la pauta de comportamiento seguida por los pacientes infantiles respecto al tratamiento (finalizar versus abandonar). Indicar, según los resultados extraídos, que la depresión de las madres es el factor relacionado más directamente con el abandono terapéutico de los niños tratados, observándose que interrumpen/rehusan el $81,8 \%$ (R.T.C. $=3,3$ ) de los pacientes cuyas madres tienen depresión.

Con respecto a la asociación entre los métodos de manejo conductual que ponen en práctica los padres de los niños estudiados y el abandono/continuidad en los tratamientos, hay que señalar que los análisis de la varianza realizados muestran diferencias significativas en cinco de las variables estudiadas al respecto, a saber: acuerdo padre/madre en normas disciplina, establecimiento de amenazas reales, utilidad normas disciplina, capacidad para establecer límites y número de actividades realizadas por los padres. La tabla 2 muestra las diferencias de medias y las $F_{(2,59)}$ significativas relativas a los métodos de manejo conductual de los padres. De esta forma, respecto a las variables "acuerdo padre/madre en normas disciplina", "establecimiento de amenazas reales" y "capacidad para establecer límites", el análisis a posteriori de Tukey reveló dos grupos claramente diferenciados, integrado uno de ellos por los padres de los niños que rehusaron el tratamiento, quiénes obtuvieron las puntuaciones significativamente más bajas en las tres variables mencionadas $(0=$ $1,25,0=1,13$ y $0=1,50$, respectivamente; $p<0,01$ ). El otro grupo quedó formado por los adultos cuyos hijos llegaron a iniciar el tratamiento, (finalizar e interrumpir) que puntuaron de forma similar (Tabla 2). 
Tabla 2. Relaclón slgnlficatlva entre los métodos de manejo conductual de los padres y la varlable finallzar - abandonar el tratamiento

\begin{tabular}{|c|c|c|c|c|c|}
\hline & & Media & Desv. TIpo & $\boldsymbol{F}$ & sig. \\
\hline $\begin{array}{l}\text { Acuerdo en } \\
\text { normas }\end{array}$ & \begin{tabular}{|l} 
Finalizan \\
Interrumpen \\
Rehúsan \\
\end{tabular} & $\begin{array}{l}3,22 \\
3,00 \\
1,25 \\
\end{array}$ & $\begin{array}{l}1,25 \\
1,12 \\
0,46 \\
\end{array}$ & 9,58 & $0,000^{* *}$ \\
\hline $\begin{array}{c}\text { Amenazas } \\
\text { reales }\end{array}$ & \begin{tabular}{|l} 
Finalizan \\
Interrumpen \\
Rehúsan \\
\end{tabular} & $\begin{array}{l}2,67 \\
3,11 \\
1,13 \\
\end{array}$ & $\begin{array}{l}1,27 \\
1,54 \\
0,35 \\
\end{array}$ & 6,38 & $0,004^{* *}$ \\
\hline $\begin{array}{c}\text { Utilidad de } \\
\text { normas/discipl. }\end{array}$ & \begin{tabular}{|l} 
Finalizan \\
Interrumpen \\
Rehúsan \\
\end{tabular} & $\begin{array}{l}2,44 \\
2,78 \\
1,50 \\
\end{array}$ & $\begin{array}{l}1,05 \\
1,56 \\
0,76 \\
\end{array}$ & 3,01 & $0,040^{\circ}$ \\
\hline $\begin{array}{c}\text { Capacidad } \\
\text { establecer } \\
\text { IIfmites }\end{array}$ & \begin{tabular}{|l} 
Finalizan \\
Interrumpen \\
Rehúsan \\
\end{tabular} & $\begin{array}{l}3,41 \\
3,56 \\
1,50 \\
\end{array}$ & $\begin{array}{l}1,08 \\
1,42 \\
0,53 \\
\end{array}$ & 10,47 & $0,000^{* *}$ \\
\hline $\begin{array}{l}\text { No Actividades } \\
\text { del padre }\end{array}$ & $\begin{array}{l}\text { Finalizan } \\
\text { Interrumpen } \\
\text { Rehúsan }\end{array}$ & $\begin{array}{l}2,09 \\
1,27 \\
1,50\end{array}$ & $\begin{array}{l}0,91 \\
0,90 \\
0,71\end{array}$ & 3,53 & $0,038^{*}$ \\
\hline
\end{tabular}

* $(p<0,05) \quad * *(p<0,01)$

En relación a la variable "utilidad normas de disciplina", las diferencias significativas se observan entre los padres de los niños que finalizaron el tratamiento $(0=2,44$; d.t. $=1,05)$ y los progenitores de los menores que rehusaron la terapia $(0=1,50$; d.t. $=0,76)$, apreciándose que la puntuación más baja corresponde, de nuevo a este último grupo $(p=0,032)$. Finalmente, respecto al factor "número de actividades realizadas por el padre", referido a las tareas conjuntas que realiza el padre con sus hijos, las diferencias significativas aparecen entre los sujetos cuyos hijos interrumpieron el tratamiento $(0=1,27$; d.t. $=0,90)$, y aquellos cuyos hijos lo finalizaron $(0=2,09$; d.t. $=0,91)$, habiendo obtenido los primeros la menor puntuación $(p=0,034)$. 
Por otro lado, la tabla 3 muestra la relación establecida entre el tipo de estrategia conductual adoptada por los padres para afrontar las situaciones difíciles y el abandono o permanencia de los niños respecto al tratamiento recomendado $\left(X^{2}{ }_{(3)}=7,905 ; p=0,046\right)$. De forma global, y previo al análisis en base a la variable continuidad/ abandono, señalar que el método de control habitualmente empleado por los padres era el castigo verbal positivo $(53,2 \%)$, seguido de la extinción $(19,3 \%)$, del razonamiento $(16,1 \%)$ y del control de estímulos $(11,3 \%)$. Por otro lado, al analizar los datos específicamente, se observa que los padres de menores que abandonaron el tratamiento no aplicaban en ningún caso extinción como método de control conductual, mientras que el $30,8 \%$ de los padres cuyos hijos finalizaron la terapia administraban usualmente este procedimiento antes del iniciar tratamiento recomendado. Respecto al empleo del razonamiento verbal o del control de estímulos, no se aprecian diferencias entre ambos grupos. Sin embargo, es en el uso del castigo positivo de naturaleza verbal donde, a juzgar por los datos obtenidos, se establecen las mayores diferencias entre ambos grupos de padres,

Tabla 3. Relación entre tlpo de estrategla conductual empleada por los padres y la varlable finallzar - abandonar el tratamiento

\begin{tabular}{|c|c|c|c|}
\hline \multirow{2}{*}{$\begin{array}{l}\text { Conductas } \\
\text { reallzadas }\end{array}$} & Abandonan & Permanecen & \multirow{2}{*}{$\frac{\text { Total }}{N}$} \\
\hline & $\%$ & $\%$ & \\
\hline \multirow{2}{*}{ Extinción } & $0 \quad 0,00$ & $12 \quad 30,8$ & 12 \\
\hline & $-2,0 * *$ & $2,0 *$ & $19,3 \%$ \\
\hline \multirow{2}{*}{ Razonam. } & 13 & 18 & 10 \\
\hline & $-0,67$ & 0,66 & $16,1 \%$ \\
\hline \multirow{2}{*}{$\begin{array}{l}\text { Control de } \\
\text { estimulos }\end{array}$} & 8,7 & 12,8 & 7 \\
\hline & $-0,13$ & 0,12 & $11,3 \%$ \\
\hline \multirow{2}{*}{$\begin{array}{l}\text { Castigo + } \\
\text { verbal }\end{array}$} & 78,3 & 38,4 & 33 \\
\hline & $2,23 *$ & $-2,23 * *$ & $53,2 \%$ \\
\hline Total & 23 & 39 & 62 \\
\hline
\end{tabular}

$\left(X^{2}=7,905 ; p=0,046\right)$

** (R.T.C. $>+/-1,96)$ 
observándose que dicha estrategia es habitualmente empleada por los progenitores cuyos hijos abandonaron el tratamiento (R.T.C.= 2.23). Añadir que se indagó, asimismo, acerca de la percepción de eficacia que tenían los padres sobre las estrategias conductuales que ellos mismos ponían en práctica, no hallándose, en este sentido, diferencias significativas entre ellos.

Se analiza a continuación cómo influyen los trastornos infantiles diagnosticados en el estado de salud de los padres y en el tipo de métodos que éstos emplean para controlar las alteraciones de sus hijos. Con este propósito se realizó un Anova tomando como variable dependiente las puntuaciones obtenidas por los progenitores en el cuestionario GHQ y como variable independiente los distintos trastomos previamente identificados (TDAH, subtipo déficit de atención por un lado, subtipos hiperactivo-impulsivo y combinado por otro y trastomo negativista desafiante). Los resultados de dicho análisis muestran cómo la puntuación de la subescala referente a "disfunción social" y el índice de salud global del cuestionario GHQ se relacionan significativamente con los trastornos estudiados en este trabajo $\left(F_{(2,59)}=5,67, p=0,006\right.$ y $F_{(2,59)}=3,46, p=0,040$, respectivamente). Los análisis a posteriori revelan que las diferencias significativas se observan entre el grupo de padres de niños hiperactivos - combinado y los grupos de padres cuyos hijos presentaban déficit de atención y trastorno negativista desafiante, mostrando los primeros una menor puntuación en ambas escalas ( $p<0,01$ ).

En este sentido, al analizar las tácticas conductuales de los padres y su ejecución real atendiendo a los trastornos detectados en los niños (Tabla 3), se observan relaciones significativas en cuatro de las variables estudiadas, a saber: Establecimiento de amenazas reales $\left(F_{(2,59)}=4,247 ; p=0,002\right)$, cumplimiento del castigo $\left(F_{(2,59)}=3,074 ; p=\right.$ $0,048)$, capacidad para establecer límites $\left(F_{(2,59)}=12,454 ; p=0,000\right)$ y el número de actividades que el padre realiza con los niños $\left(F_{(2,59)}=\right.$ $3,678 ; p=0,034)$. En el análisis a posteriori se comprueba que, en todos los casos, la menor puntuación obtenida por los padres de los niños con trastorno hiperactivo - combinado marca significativamente el límite inferior $(p<0,01)$. Asi, en el caso de las variables establecimiento de amenazas reales y capacidad para establecer límites, las diferencias se establecen con los otros dos grupos de padres $(p<0,01)$. Respecto 
a la ejecución real de órdenes paternas, son los progenitores de niños con déficit de atención quienes logran mayor nivel de aplicación $(p<$ 0,01 ), mientras que los padres de niños con trastorno negativista desafiante realizan mayor número de actividades con sus hijos $(p<0,05)$.

Tabla 4. Relaclón entre métodos de manejo conductual adoptados por los padres y el trastorno infantil diagnosticado

\begin{tabular}{|c|c|c|c|c|c|}
\hline & & Media & Desv. $T$. & $F$ & Sig. \\
\hline \multirow{3}{*}{$\begin{array}{c}\text { Amenazas } \\
\text { reales }\end{array}$} & DA & 3,08 & 1,24 & & \\
\hline & $\mathrm{HI}-\mathrm{COM}$ & 1,70 & 0,82 & 4,247 & $0,002^{* *}$ \\
\hline & TND & 2,84 & 1,30 & & \\
\hline \multirow{3}{*}{$\begin{array}{l}\text { Cumplimiento } \\
\text { de castigos }\end{array}$} & DA & 3,25 & 1,22 & & \\
\hline & $\mathrm{HI}-\mathrm{COM}$ & 2,00 & 0,94 & 3,074 & $0,048^{*}$ \\
\hline & TND & 2,79 & 1,27 & & \\
\hline \multirow{3}{*}{$\begin{array}{l}\text { Capacidad } \\
\text { establecer } \\
\text { límites }\end{array}$} & DA & 3,83 & 1,11 & & \\
\hline & $\mathrm{HI}-\mathrm{COM}$ & 1,80 & 0,63 & 12,454 & $0,000^{* *}$ \\
\hline & TND & 3,37 & 1,07 & & \\
\hline \multirow{3}{*}{$\begin{array}{c}N^{e} \\
\text { Actividades } \\
\text { del padre }\end{array}$} & DA & 1,77 & 0,92 & & \\
\hline & $\mathrm{HI}-\mathrm{COM}$ & 1,22 & 0,83 & 3,678 & $0,034^{*}$ \\
\hline & TND & 2,16 & 0,91 & & \\
\hline
\end{tabular}

$*(p<0,05) \quad * *(p<0,01)$

Por último, se analiza la percepción de eficacia que mantienen los padres respecto a las estrategias conductuales que ellos aplican mediante un Anova, tomando como factor el diagnóstico de los niños. En este caso, resultan significativas las diferencias presentes tanto en la percepción de eficacia de las madres $\left(F_{(2,58)}=4,052 ; p=0,027\right)$ como de los padres $\left(F_{(2,58)}=3,244 ; p=0,042\right)$. Los análisis a posteriori muestran que estas diferencias se observan entre el grupo de padres con hijos diagnosticados con trastorno hiperactivo atencional (con mayor puntuación), y el grupo de progenitores con hijos diagnosticados con trastorno hiperactivo - combinado $(p=0,027)$. En sintesis, los padres de los niños con déficit de atención son los que mayor percepción de eficacia tienen en relación a las tácticas conductuales 
que emplean para controlar las alteraciones de sus hijos, siendo los padres de los niños hiperactivos y tipo combinado los que perciben como ineficaces sus métodos de control.

\section{DISCUSIÓN}

Los resultados obtenidos en este trabajo respecto a la tasa de abandonos terapéuticos concuerdan con los hallazgos de investigaciones previas realizadas en el ámbito infantil. El $37 \%$ de los pacientes infantiles diagnosticados previamente con trastorno hiperactivo y negativista desafiante abandonaron el tratamiento, porcentaje similar al obtenido por Kazdin y Wassell (1998). Así pues, la incidencia de esta cuestión, respecto a estos trastornos, se encuentra en los límites ya señalados en otros estudios sobre el tema.

Asimismo, queda de manifiesto que el trastorno diagnosticado influye significativamente en la continuidad o abandono del tratamiento infantil recomendado. Es claro que los menores con Trastorno Negativista Desafiante se distancian de los niños con trastorno hiperactivo, pues más del $85 \%$ finalizaron la intervención recomendada tras el diagnóstico. Es probable que la gravedad y notoriedad de las alteraciones caracteristicas (desafío, hostilidad, desobediencia, etc.) unido, por un lado, a las experiencias fallidas de los padres cuando por sí solos han intentado controlar el comportamiento de su hijo y, por otro, a la modalidad de tratamiento administrada, menos compleja que otras alternativas en cuanto a condiciones de aplicación y más breve en tiempo de ejecución, hayan contribuido, entre otros factores, a asegurar la permanencia de los niños en el tratamiento administrado.

En cuanto a las variables familiares investigadas hay que añadir, respecto a la salud general percibida por los adultos, que los padres de niños que interrumpieron el tratamiento mostraban mayor índice de problemas sociales en comparación con los progenitores cuyos hijos rehusaron acudir a la primera sesión terapéutica. Asimismo, resulta notorio, teniendo en cuenta las características del trastorno, que los padres de los pacientes diagnosticados con los subtipos hiperactivos y combinados mostraran menor grado de disfunción social, e incluso, mejor estado de salud general. Es posible que esta circunstancia responda en alguna medida a la propia estructura y configuración del 
cuestionario empleado. Este instrumento muestra escasa sensibilidad cuando se trata de valorar el estado de salud de individuos que sufren trastornos de larga duración, pues los afectados tienden a responder "igual que lo habitual" ante síntomas y problemas que experimentan de forma crónica (Goldberg, 1978). Teniendo en cuenta que esta prueba (GHQ-28) indaga sobre el estado de salud percibido en las dos últimas semanas, es probable que los padres de niños con trastomo hiperactivo, habituados desde hace tiempo a las alteraciones y problemas que muestran sus hijos, no perciban cambios sustanciales en su funcionamiento social una vez que han decidido demandar ayuda clínica.

Asimismo, respecto a la incidencia que la depresión de las madres ejerce en los abandonos terapéuticos registrados en este trabajo, hemos de aludir al trabajo de Lesesne, Visser y White (2003) en el se constata una asociación cuatro veces superior entre madres con depresión e hijos diagnosticados de TDAH respecto a población normal.

En cuanto a los métodos de manejo conductual adoptados por los adultos que han participado en el trabajo y su influencia en las variables estudiadas añadir que los padres de los niños que rehusaron el tratamiento, mostraban, comparativamente, menos recursos y habilidades para manejar la conducta de sus hijos que los progenitores cuyos hijos sí iniciaron la terapia. Destaca el escaso acuerdo entre ambos miembros de la pareja para establecer normas, al tiempo que distan de los otros grupos al plantear amenazas reales que puedan llevarse a efecto, quedando, asimismo, de manifiesto sus dificultades para establecer límites conductuales. Sin duda, tales circunstancias influyen en la percepción que este grupo de progenitores mantiene acerca de la limitada utilidad de los castigos que administran. De este modo, y en lo relativo a las iniciativas de control conductual, los padres cuyos hijos no llegaron a iniciar la intervención se distancian de aquellos con hijos que si comenzaron el tratamiento.

Al analizar las actividades conjuntas (juegos compartidos, tareas escolares, etc.) realizadas por ambos progenitores con el niño, se constata que los padres cuyos hijos bien abandonaron el tratamiento o bien rehusaron $\mathrm{e}$ interrumpieron, interactuaban comparativamente menos con sus hijos. Es probable que, debido a las características propias del trastorno hiperactivo y a las exigencias de afrontamiento 
que éste requiere por parte de los adultos, las actividades conjuntas a realizar entre padres e hijo constituyan una fuente continúa de estrés de manera que los adultos tiendan a evitarlas, en consonancia con los planteamientos de Pelechano (2000) cuando se refiere a los acontecimientos cotidianos negativos y sus repercusiones en el estado de salud de los adultos.

Respecto a los métodos conductuales adoptados habitualmente por los adultos para hacer frente a las alteraciones de comportamiento que presentan los menores estudiados, los datos muestran diferencias que se relacionan con la permanencia o abandono terapéutico. Los padres que aseguran a sus hijos en la terapia recurren sobre todo a extinción a diferencia de los progenitores cuyos hijos abandonaron, que emplean mayoritariamente castigo verbal positivo. Si se tienen en cuenta los resultados anteriores parece claro que las dificultades que estos padres muestran para establecer límites conductuales, acordar normas, plantear amenazas reales, etc. les instan a recurrir prioritariamente al castigo. De esta manera la conducta disruptiva de su hijo queda controlada, al menos de forma inmediata, aunque dado el escaso mantenimiento de los cambios conductuales que esta táctica lleva consigo, les induzca a repetir y reiterar idéntico método una y otra vez.

En relación a la influencia del trastorno infantil diagnosticado en la salud general de los padres, en los métodos conductuales que ponen en práctica para manejar el comportamiento de sus hijos y en la percepción de eficacia que tienen sobre dicho métodos, se constata que los progenitores de niños diagnosticados con trastorno negativista desafiante muestran mayor disfunción social, indicando que, en los padres, este trastorno, más específico en cuanto áreas de afectación, influye de manera definida en el funcionamiento y adaptación social. Asimismo, la influencia desfavorable de la hiperactividad se extiende hacia las habilidades y recursos conductuales que adoptan los progenitores, pues los padres de niños hiperactivos presentan más dificultades para llevar a la práctica tácticas de control conductual y realizan menos actividades conjuntamente con sus hijos, al tiempo que también perciben como mas ineficaces sus propios métodos.

En síntesis, los datos obtenidos indican que el trastorno hiperactivo afecta negativamente a los padres que ponen en práctica métodos y tácticas de control conductual percibidos por ellos mismos como 
ineficaces, coincidiendo en este sentido con las aportaciones de otros estudios sobre el tema (Lesesne et al., 2003). No obstante, respecto al estado de salud general de los progenitores, se requieren futuros estudios que empleen otros instrumentos más específicos.

\section{REFERENCIAS BIBLIOGRÁFICAS}

American Psychiatric Association (2000). DSM-IV-TR. Manual diagnóstico y estadistico de los trastornos mentales. Barcelona: Masson, (trad. Cast. 2002).

Armbruster, P. y Kazdin, A.E. (1994). Attrition in child psychotherapy. Advances in Clinical Child Psychology, 1994; 16, 81-108.

Barkley, R. A. (1997). Niños desafiantes: materiales de evaluación y folletos para los padres $2^{a}$ edic. New York: Guilford Press, 1997 (trad. por J. J. Baumeister y cols.).

Barkley, R. A., Murphy, K. R., y Bauermeister, J. J. (1998). Trastomo por déficit de atención e hiperactividad. Un manual de trabajo clínico. Nueva York: Guilford Press.

Beck, A. T., Epstein, N., Brown, G, y Steer, R. A. (1988). An inventory for measuring clinical anxiety: Psychometric properties. Joumal of Consulting and Clinical Psychology, 56(6), 893-897.

Beck, A. T., Ward, C.H., Mendelson, M., Mock, J., y Erbaugh, J. (1961). An inventory for measuring depression. Archives of General Psychiatry, 4, 561-571.

Brown, T. E. (2003). Trastornos por déficit de atención y comorbilidades en niños, adolescentes y adultos. Barcelona: Masson. (Orig. 2000) Collett, B. R., Ohan, J. L. y Myers, K. M. (2003). Ten-year review of rating scales. VI: Scales assessing externalising behaviors. Journal of American Academy of Child and Adolescent Psychiatry, 42(10), 1143-1170.

Corcoran, J. e Ivery, J. (2004). Parent and child attributions for child behavior: Distinguishing factors for engagement and outcome. Families in Society, 1, 101-106.

Corkum, P., Rimer, P. y Schachar, R. (1999). Parental knowledge of attention-deficit hyperactivity disorder and opinions of treatment options: Impact on enrolment and adherence to a 12-month treatment trial. Canadian of Journal Psychiatric, 44(10): 1043-1048. 
DuPaul, G. J., Power, T. J., Anastopoulos, A. D., y Reid, R. (1998). ADHD Rating Scale-IV: Checklists, Norms, and Clinical Interpretation. New York: Guilford Press.

Garfield, S. L. (1989). Giving up on child psychotherapy: Who drops out? Comment on Weisz, Weiss and Langmeyer. Journal of Consulting and Clinical Psychology, 1989; 57(1) 168-169.

Garcia, C. R. (1999). Manual para la utilización del cuestionario de salud general de goldberg. Adaptación cubana. Revista Cubana de Medicina General Integral, 15(1), 88-97.

Goldberg, D. (1978). Manual del general health questionnaire. Windsor:

NFER Publishing.

Jones, M. L. Eyberg, S. M., Adams, C. D. y Boggs, S. R. (1998).

Treatment acceptability of behavioural interventions for children: An assessment by mothers of children with disruptive behavior disorders.

Child and Family Behavior Therapy, 20(4), 15-26.

Kazdin, A. E. (1996). Dropping out of child psychotherapy: Issues for research and implications for practice. Clinical Child Psychology Psychiatry, 1, 133-156.

Kazdin, A. E. (2003). Psychotherapy for children and adolescents. Annual Review of Psychology, 54, 253-276.

Kazdin, A. E. y Mazurick, J. (1994). Dropping out of child psychotherapy: Distinguishing early and late dropouts over the course of treatment. Joumal of Consulting and Clinical Psychology, 62(5), 1069-1074. Kazdin, A. E. y Wassell, G. (1998). Treatment completion and therapeutic change among children referred for outpatient therapy. Professional Psychology, Research and Practice, 29(4), 332 340.

Kazdin, A. E., Holland, L. y Crowley, M. (1997). Family experience of barriers to treatment and premature termination from child therapy. Joumal of Consulting and Clinical Psychology, 65(3), 453-463.

Kazdin, A. E., Siegel, T. C. y Bass, D. (1990). Drawing on clinical practice to inform research on child and adolescent psychotherapy: Survey of practitioners. Professional Psychology, Research and Practice, 21(3), 189-198.

Kraim, A. L. (2004). The role of parent and child perceptions of readiness for change, problem severity, and treatment acceptability in the pursuit of treatment for attention-deficit/hyperactivity disorder. Dissertation 
Abstracts International: Section-B:-The-Sciences-and-Engineering, 64(7-B), 3530.

Levitt, E. E. (1971). Research on psychotherapy with children. En A. E. Bergin y S. L. Garfield (Eds.) Handbook of psychotherapy and behavior change. New York: Wiley.

Lesesne, C. A., Visser, S. N. y White, C. P. (2003). With matemal mental health and use of health care resources attention-deficithyperactivity disorder in school-aged children: association. Pediatrics, 111, 1232-1237. Lynn, A. (2004). The role of parent and child perceptions of readiness for change, problem severity and treatment acceptability in the pursuit of treatment for attention-deficit/hyperactivity disorder. Dissertation Abstract International: Sec B: Science Engineer, 64(7-B) 3530.

Moreno, G. I. (2005). Características de la intervención terapéutica en la infancia. En I. Comeche y M. A. Vallejo (Eds.). Manual de terapia de conducta en la infancia, (PP. 25-68). Madrid: Dykinson.

Osman, A., Hoffman, J., Barrios, F. X., Kopper, B. A., Breitenstein, J. L., y Hahn, S. K. (2002). Factor structure, reliability, and validity of the Beck Anxiety Inventory in adolescent psychiatric inpatients. Journal of Clinical Psychology, 58(4), 443-456.

Pekarik, G. (1991). Relationship of expected and actual treatment duration for child and adult clients. Joumal of Consulting and Clinical Psychology, 20, 121-125.

Pekarik, G. y Stephenson, L. A. (1988). Adult and child client differences in dropout research. Journal of Consulting and Clinical Psychology, 17, 316-321.

Pelechano, V. (2000). Psicología sistemática de la personalidad. Barcelona: Ariel.

Pelham, W. y Murphy, H. A. (1986). Attention deficit and conducts disorder. En H. Hersen (Ed.) Pharmacological and behavioral treatments: An integrative approach. New York: Wiley, 1986.

Power, T. J., Costigan, T. E., Eiraldi, R. B. y Leff, S. S. (2004). Variations in Anxiety and Depression as a Function of ADHD Subtypes Defined by DSM-IV: Do Subtype Differences Exist or Not? Journal of Abnormal Child Psychology, 32(1), 27-37.

Remschmidt, H. (2003). Evidence concerning the effectiveness of psychotherapies with children and adolescents. Current Opinion in Psychiatry, 16(4), 389-393. 
Salvador, L. y Melgarejo, M. (2000). Cumplimiento terapéutico. Barcelona: Ars Médica.

Sanz, J. y Navarro, E. (2003). Propiedades psicométricas de una nueva versión española del inventario de ansiedad de Beck (BAI) en estudiantes universitarios. Ansiedad y Estrés, 9(2), 59-84.

Servera, M.; Bornas, X.; Moreno, I. (2001). Hiperactividad infantil: conceptualización, evaluación y tratamiento. En V. E. Caballo y $\mathrm{M}$. A. Simón (dirs.), Manual de psicología clínica infantil y del adolescente (pp. 401-433). Madrid: Pirámide.

Vázquez, C. y Sanz, J. (1997). Fiabilidad y valores normativos de la versión española del inventario para la depresión de Beck de 1978. Clínica y Salud, 3, 403-423.

Webster-Stratton, C. y Hammond, M. (1990). Predictors of treatment outcome in parent training for families with conduct problem children. Behavior Therapy, 21, 319-337.

Wierzbicki, M. y Pekarik, G. A (1993). Meta-analysis of psychotherapy dropout. Professional Psychology, Research And Practice, 24(2): 190-195. 